\title{
A MODIFIED ADAPTIVE WAVELET SHRINKAGE SPECKLE FILTER FOR ULTRASOUND IMAGES
}

\author{
${ }^{1}$ Nirmaladevi Periyasamy and ${ }^{2}$ Asokan Ramasamy \\ ${ }^{1}$ Department of Electronics and Communication Engineering, \\ Kongu Engineering College, Perundurai, Erode, Tamil Nadu, India \\ ${ }^{2}$ Kongunadu College of Engineering and Technology, Thottiyam, Tamil Nadu, India
}

Received 2013-06-17, Revised 2013-09-11; Accepted 2013-09-16

\begin{abstract}
The usage of ultrasound imaging for medical diagnosis is limited due to the presence of speckle noise. In this study Modified Adaptive Wavelet Shrinkage Filter (MAWSF) in the translational invariant domain is proposed for the removal of speckle noise. The adaptive wavelet threshold function removes the fixed bias of soft thresholding. A new inter-scale dependency model is proposed, to perform a primary clustering of signal of interest and noise. Then, anew sub-band adaptive threshold is determined for all high frequency sub-bands at various decomposition levels, to shrink the noisy coefficients. Experiment is conducted on several ultrasound scan images. The results show that this method yields better visual quality and Peak Signal to Noise Ratio (PSNR). Improvement in preservation of edge details is also found measured with Edge Preservation Index (EPI) measure.
\end{abstract}

Keywords: Stationary Wavelet Transform, Inter and Intra Scale Dependencies, Adaptive Threshold Function, Image Denoising

\section{INTRODUCTION}

Digital Images are contaminated by noise during acquisition or transmission stages. Speckle noise is a kind of multiplicative noise. Synthetic Aperture Radar (SAR), Medical Ultrasound images are said to contain speckle noise. Speckle noise results due to the constructive-destructive interference of the coherent ultrasound pulses that are backscattered from the tiny biological tissues. Ultrasoaund images are formed by transmitting a sound wave and receiving the echoes that are reflected from the biological tissues, give rise to a granular pattern in the imaging data. This may reduce the contrast and resolution of the ultrasound image and hence it becomes a tedious process for the physician to make a diagnosis. The presence of speckle degrades the quality of images and they become unsuitable for further processing. Hence, image denoising is a preprocessing step in any image processing algorithm. The main objective of image denoising is (i) to remove the unwanted noise present in the images (ii) to preserve the edges and fine details (iii) to improve the visual quality of the images.

Recent investigations in the literature, (Kaur et al., 2011) and references therein show a significant use of wavelet transform for denoising, called non linear filtering. Wavelet denoising attempts to remove noise and preserve the signal details irrespective of its frequency content.

Thresholding is a non-linear technique, which can operate on one wavelet coefficient at a time. In thresholding, the coefficient is smaller than the threshold are made zero or shrunk towards zero otherwise it is kept or modified. The standard thresholding methods are hard thresholding and soft thresholding. Soft thresholding preserves the smoothness and fixed bias is also present.

Shift invariant wavelet denoising is better than discrete wavelet shrinkage denoising (Yang et al., 2011). The Stationary Wavelet Transform (SWT) overcomes

Corresponding Author: Nirmaladevi Periyasamy, Department of Electronics and Communication Engineering, Kongu Engineering College, Perundurai, Erode, Tamil Nadu, India 
the lack of translation-invariance property of the discrete wavelet transform by removing the down samplers and up samplers in the wavelet transform. SWT is a redundant scheme as the output of each level has the same number of samples as the input. Small image details can be revealed in finer scales and it can be determined by inter-scale dependencies which involves two adjacent scales. Sudha et al. (2009) and Alavandan and Baboo (2012); exploited alternate methods of the dependencies among the wavelet coefficients for the removal of noise. Dwivedi and Singh (2010) proposed a new interscale dependency measure to enhance the power quality events. A variation of their approach is proposed in this study. The classification of signal of interest and noise pixels is done at a preliminary level, using this proposed inter scale dependency measure. This found to improve the preservation of edge information.

Kalavathy and Suresh (2011) proposed an adaptive thresholding function based on the similarity between the pixels. Hussain and Gorashi (2012) proposed a new hybrid thresholding function using a hybrid and wavelet algorithms and achieved an improved performance. Anand et al. (2011) used an improved threshold in the undecimated wavelet domain for speckle suppression. Qin et al. (2010) developed an improved thresholding funcion to reduce the fixed-bias of the soft thresholding technique. An enhanced approach for their adaptive thresholding function is developed in this study. The denoising performance is enhanced by utilizing the dependencies among the wavelet coefficients at different scales by using stationary wavelet transform and by the selection of a new sub-band adaptive threshold for shrinking the wavelet coefficients. The performance measures like Peak Signal to Noise ratio (PSNR), Mean Square Error (MSE), Structural Similarity Index Measure (SSIM), Equivalent Number of Looks (ENL), Edge Preservation Index (EPI) and visual quality are found, to compare the performance the proposed approach.

This study includes following sections. Section 2 explains the materials and methods for the proposed MAWSF, Section 3 shows the results of the experiment and Section 4 discusses the performance improvement of the proposed method over the existing techniques.

\section{MATERIALS AND METHODS}

Information about the signal of interest and noise could be better studied by determining the secondary wavelet transform properties. They are the intra and inter-scale dependencies among the wavelet coefficients. The dependency between the coefficients of the same sub band is called intra-scale dependency and dependency existing between the sub bandsin adjacent scales is called inter-scale dependency. Figure 1 shows one level decomposition of stationary wavelet transform. It is an undecimated wavelet transform which preserves the shift invariance property.

In Fig. 2, $\mathrm{j}$ is the finest scale and $\mathrm{j}+1$ is the coarsest scale of wavelet decomposition. Coarsest scale coefficients are parent coefficients and finest scale coefficients are child coefficients. If the magnitude of parent coefficient is small then the magnitude of child coefficient must be small. This property of wavelet coefficients is used as a measure of homogenous regions in the images. The large magnitude wavelet coefficients produced at finer scales are more likely to yield significant parents at coarser scales. However, the coefficients caused by noise would decay rapidly along scales. Also, estimating the data locally by using the correlation of wavelet coefficient in the local neighbourhoodhas been widely used in the denoising algorithms. Through the literature it is found that intra scale and inter scale dependencies exist among wavelet coefficients within and across the wavelet decomposition scales (i.e., the wavelet coefficients are not only correlated with their neighbours in a sub-band, but also the adjacent wavelet scales are strongly correlated in general). Therefore, wavelet intra and inter scale dependency information have been combined in the proposed method to improve the performance of restoration.

\subsection{Wavelet Thresholding Algorithm}

Wavelet based denoising technique performs noise removal in three steps. (i) Decomposing the image into various sub-bands at various levels (ii) Shrink the wavelet coefficients using soft or hard thresholding function (iii) Reconstruct the restored image by applying inverse wavelet transform.

\subsubsection{Thresholding Functions}

In wavelet domain, large magnitude coefficients are said to contain important image features and small magnitude coefficients are associated with noise. Therefore a suitable threshold is applied to remove noise, by making the small magnitude coefficients to zero and scaling the large magnitude coefficients towards zero. There are two types of traditional thresholding functions. There are two types of traditional thresholding functions. 


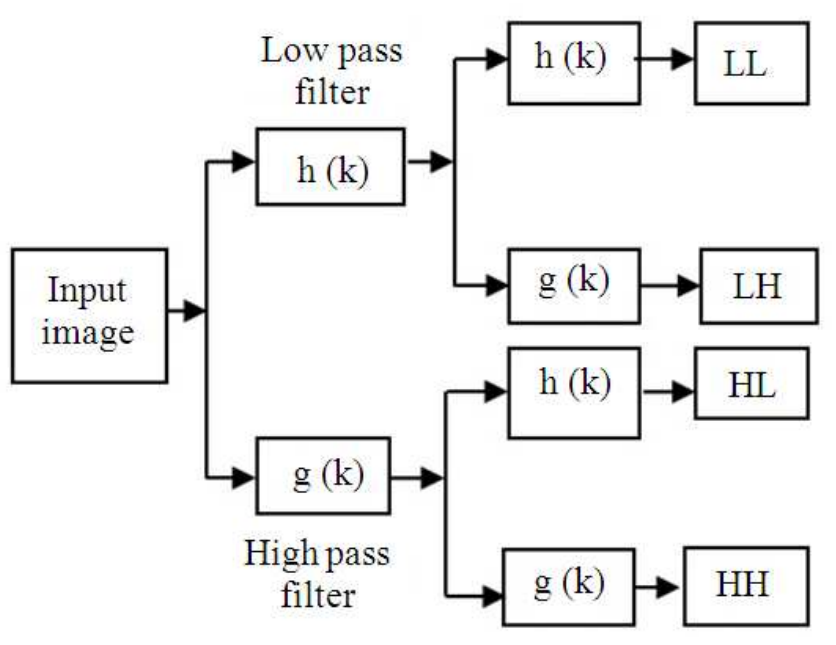

Fig. 1. One level decomposition of stationary wavelet transform

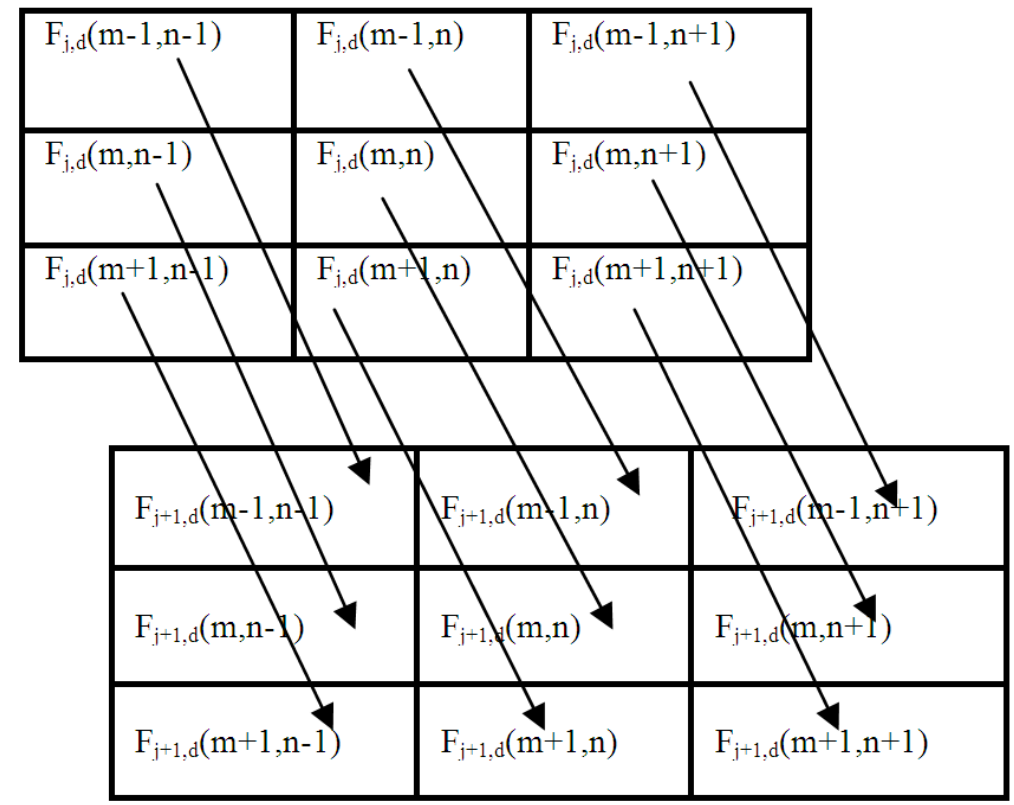

Fig. 2. Inter scale and Intra scale dependency among adjacent level coefficients

\subsubsection{Soft Thresholding Function Equation (1)}

$\widehat{\mathrm{W}}_{\mathrm{I}, \mathrm{J}}=\operatorname{sgn}\left(\mid \mathrm{W}_{\mathrm{i}, \mathrm{j}}\right)\left(\left|\mathrm{W}_{\mathrm{i}, \mathrm{j}}\right|-\lambda\right)\left|\mathrm{W}_{\mathrm{i}, \mathrm{j}}\right| \geq \lambda=0\left|\mathrm{~W}_{\mathrm{i}, \mathrm{j}}\right|<\lambda$

\subsubsection{Hard Thresholding Function Equation (2)}

$\widehat{\mathrm{W}}_{\mathrm{I}, \mathrm{J}}=\mathrm{W}_{\mathrm{i}, \mathrm{j}}\left|\mathrm{W}_{\mathrm{i}, \mathrm{j}}\right| \geq \lambda=0\left|\mathrm{~W}_{\mathrm{i}, \mathrm{j}}\right|<\lambda$
Where:

$\mathrm{W}_{\mathrm{j}, \mathrm{k}}=$ Stands for the wavelet coefficients

$\lambda=$ Stands for the threshold value

$\widehat{\mathrm{W}}_{\mathrm{Jk}}=$ Stands for the restored wavelet coefficients

\subsection{Proposed Thresholding Algorithm}

In the traditional hard thresholding method, the wavelet coefficient processed by the threshold value 
have discontinuous point on the threshold $\lambda$ and $-\lambda$, which may cause Gibbs shock to the useful reconstructed signal. In soft-thresholding method, continuity is good, but it introduces a constant bias between the processed and the original wavelet coefficient. Hence, the original features of the image cannot be maintained effectively.

The performance of soft thresholding is improved by reducing the fixed bias. Hence, the coefficients are estimated between $\left|W_{i, j}\right|-\lambda$ and $W_{i, j}$. Therefore a weighted method is used to construct the new adaptive threshold function defined by (Qin et al., 2010) as follows Equation (3):

$$
\begin{aligned}
& \widehat{\mathrm{W}}_{\mathrm{I}, \mathrm{J}}=\operatorname{sgn}\left(\mathrm{W}_{\mathrm{i}, \mathrm{j}}\right)\left(\left|\mathrm{W}_{\mathrm{i}, \mathrm{j}}\right|-\left(1-\exp \left(\frac{-\mathrm{m}}{\left|\mathrm{W}_{\mathrm{i}, \mathrm{j}}\right|^{2}-\lambda_{\mathrm{j}}^{2}}\right)\right) \lambda_{\mathrm{j}}\right. \\
& \text { for }\left|\mathrm{W}_{\mathrm{i}, \mathrm{j}}\right| \geq \lambda_{\mathrm{j}}=0 \text { for }\left|\mathrm{w}_{\mathrm{i}, \mathrm{j}}\right|<\lambda_{\mathrm{j}}
\end{aligned}
$$

where, $\mathrm{M}$ is a positive constant. When $\mathrm{m} \rightarrow \infty$, the thresholding function approaches the soft thresholding and when $\mathrm{m} \rightarrow 0$ it approaches hard thresholding function.

\subsection{Parameters for Thresholding}

The optimal selection of the threshold $\lambda$ yields a better denoising performance. If the threshold $\lambda$ is a constant parameter then it will result in too many kill of the wavelet coefficients. Therefore $\lambda$ for the adaptive threshold function is selected as subband adaptive exploiting inter and intra scale dependencies.

The subband adaptive threshold is given by Equation (4):

$$
\lambda_{\mathrm{j}}=\beta \mathrm{T}_{\text {sub }}
$$

where, $\beta$ is a subband adaptation parameter given by Equation (5):

$$
\beta=\sqrt{\frac{\log M}{2^{j}}}
$$

Here $\mathrm{M}$ is the total number of coefficients and $\mathrm{j}$ is the current level of decomposition.

\subsubsection{Intra Scale Dependency Model}

Wavelet coefficient dependencies exist within and also across the sub bands. Small magnitude coefficients at coarser scales yield insignificant children at finer scale. The coefficients that are affected by noise decay rapidly across scales. Signal variance is estimated using the intra dependency of the wavelet coefficients in a subband using local neighborhood.

The threshold $\mathrm{T}_{\mathrm{B}}$ is defined as Equation (6):

$$
\mathrm{T}_{\text {sub }}=\left\{\begin{array}{cc}
\frac{\hat{\sigma}_{\mathrm{n}}^{2}}{\hat{\sigma}_{\mathrm{x}}}, & \hat{\sigma}_{\mathrm{n}}^{2}<\hat{\sigma} \mathrm{x}^{2} \\
\max \left(\left|\mathrm{Y}_{\mathrm{k}}\right|\right), & \hat{\sigma}_{\mathrm{n}}^{2} \geq \hat{\sigma} \mathrm{x}^{2}
\end{array}\right.
$$

Noise variance $\hat{\sigma}_{\mathrm{n}}^{2}$ is estimated as follows Equation (7):

$$
\hat{\sigma}_{\mathrm{n}}^{2}=\left(\frac{\operatorname{median}\left(\left|\mathrm{Y}_{1}\right|\right)}{0.6745}\right)^{2}
$$

Where:

$\mathrm{Y}_{\mathrm{i}}=$ Belongs to subband $\mathrm{HH} 1$

$\sigma_{\mathrm{X}}{ }^{2}=$ The signal variance

The estimation of data locally is the most widely used methodology in image denoising algorithms. Each wavelet coefficient is modeled as a mixture of Generalized Gaussian Distribution variables with unknown slowly spatially varying parameters. The estimation of these parameters is carried out as a function of its neighbouring coefficients.

The signal variance $\hat{\sigma}_{\mathrm{x}}^{2}$ can be estimated using the standard deviation given below Equation (8):

$\hat{\sigma}_{\mathrm{X}}=\sqrt{\max \left(\hat{\sigma}_{\mathrm{y}}^{2}-\hat{\sigma}_{\mathrm{n}}^{2}, 0\right)}$

where, $\hat{\sigma}_{y}^{2}=\frac{2}{N_{s}} \sum_{n=1}^{N s} Y_{n}^{2}, N_{s}$ is the no. of coefficients in the sub-band.

Thus the estimation of signal threshold exploits the intra scale dependency of wavelet coefficients in the sub-bands.

\subsubsection{Inter scale Dependency Model}

In this study a new inter scale dependency model is proposed Equation (9): 


$$
\mathrm{W}_{\mathrm{j}}(\mathrm{m})=0 \text { if } \mathrm{W}_{\mathrm{j}+1}\left(\frac{\mathrm{m}}{2}\right) \leq \mathrm{t}_{\mathrm{j}}
$$

For the coarsest scale $\mathrm{j}+1$ of decomposition, define a weight matrix $U_{i}$ with all entries 1 and multiply with wavelet coefficients.

For the finest scale $\mathrm{j}$ of decomposition define a weight matrix $U_{i}$ with zero entries where the coefficients in the $j+1$ level are above the adaptive threshold $t_{j}$ and with entries as 1 otherwise. Multiply with the corresponding finest scale wavelet coefficients.

The adaptive threshold $t_{j}$ is defined as Equation (10):

$\mathrm{t}=\alpha \cdot \hat{\sigma}_{\mathrm{n}}$

where, $\alpha$ is the positive constant having the value 2 .

The signal variance is then estimated as given in Equation (8). Thus the variance estimated for finest scale now exploits the inter scale dependency of wavelet coefficients.

\subsection{Algorithm}

The following steps are to be processed for the noise removal in ultrasound images:

Step 1: Decomposition of the input noisy image to $\mathbf{J}$ levels using stationary wavelet transform.

Step 2: Estimation of noise variance using Equation (7)

Step 3: Estimation of the signal variance for all the subbands at different levels exploiting inter and intra scale dependency models using Equation (8)

Step 4: Estimation of the threshold using Equation (6)

Step 5: Apply the new adaptive thresholding function to shrink the wavelet coefficients

Step 6: Application of inverse stationary wavelet transform to get the denoised image

\section{RESULTS}

Experiments were conducted on several ultrasound scan images for different noise variances. Several performance measures given from Equation (11) to Equation (15) are measured Equation (12 to 14):

$$
\text { PSNR }=10 \log _{10}\left[\frac{255^{2}}{\mathrm{MSE}}\right] \mathrm{dB}
$$

$$
\begin{aligned}
& \text { MSE }=\frac{1}{\mathrm{MN}} \sum_{\mathrm{i}=1}^{\mathrm{M}} \sum_{\mathrm{j}=1}^{\mathrm{N}}(\mathrm{X}(\mathrm{i}, \mathrm{j})-\mathrm{P}(\mathrm{i}, \mathrm{j}))^{2} \\
& \mathrm{ENL}=\frac{1}{\mathrm{H}} \sum_{\mathrm{i}=1}^{\mathrm{H}} \frac{\mu^{2}}{\sigma^{2}} \\
& \operatorname{SSIM}(\mathrm{x}, \mathrm{y})=\frac{\left(2 \mu_{\mathrm{x}} \mu_{\mathrm{y}}+\mathrm{C}_{1}\right)\left(2 \sigma_{\mathrm{xy}}+\mathrm{C}_{2}\right)}{\left(\mu_{\mathrm{x}}^{2}+\mu_{\mathrm{y}}^{2}+\mathrm{C}_{1}\right)\left(\sigma_{\mathrm{x}}^{2}+\sigma_{\mathrm{y}}^{2}+\mathrm{C}_{2}\right)} \\
& \operatorname{EPI}=\frac{\sum\left(\nabla \mathrm{I}\left(\mathrm{x}_{\mathrm{i}}-\overline{\nabla \mathrm{I}}\right)\right)\left(\overline{\nabla \hat{\mathrm{I}}} \mathrm{x}_{\mathrm{i}}-\overline{\nabla \hat{\mathrm{I}}}\right)}{\sqrt{\sum}\left(\nabla \mathrm{I}\left(\mathrm{x}_{\mathrm{i}}-\overline{\nabla \mathrm{I}}\right)\right)^{2} \sum\left(\overline{\nabla \hat{\mathrm{I}}} \mathrm{x}_{\mathrm{i}}-\overline{\nabla \hat{\mathrm{I}}}\right)^{2}}
\end{aligned}
$$

where, $X(i, j)$ Original Image, $P(i, j)$ denoised Image, $\mu_{x}$ mean of original Image, $\mu_{\mathrm{y}}$ mean of denoised Image, $\sigma_{x}^{2}$ variance of original image, $\sigma_{y}^{2}$ variance of denoised image. $2 \sigma_{\mathrm{xy}}$ is the covariance of $\mathrm{x}$ and $\mathrm{y} \mathrm{C}_{1}=\left(\mathrm{K}_{1}, \mathrm{~L}\right)^{2}, \mathrm{C}_{2}=$ $\left(\mathrm{k}_{2}, \mathrm{~L}\right)^{2} ; \mathrm{K}_{1}=0.01, \mathrm{~K}_{2} 0.03$. $\mathrm{L}$ is the dynamic range of pixels.

The performance of the proposed filter is tested for lena image with synthesized Gaussian noise of variance 0.02 , in the translation invariant domain. PSNR value is found to be improved than wavelet domain soft thresholding approach as given in Table 1. An improvement of $4.5 \mathrm{~dB}$ is seen, for noise removal in the translation invariant wavelet domain.

The experimental setup used in this study is, intel i3 processor with 4GB RAM. MATLAB version $\mathrm{R} 2009 \mathrm{~b}$ is used for simulation. For testing,ultrasound images were used.The test images are obtained from Edapal Hospital, Kerala. A database with 50 images are obtained from the scan centre. The images are of dimension $512 \times 512$. Speckle noise with noise variances $0.01,0.04,0.08$ and 0.1 are added to the ultrasound images and tested. Figure 3 shows the Ultrasound fetal image used in this experiment.

Table 2 shows the performance comparison of the proposed filter for various noise variances. Figure 4-7 show the visual quality improvement for different noise variances.

Comparison measures of the proposed approach (MAWSF) are taken by with soft thresholding approach, new subband adaptive threshold with bayes threshold.

Table 1. Comparison of lena image

\begin{tabular}{lll}
\hline PSNR & Wavelet & $\begin{array}{l}\text { Translation invariant } \\
\text { wavelet domain }\end{array}$ \\
\hline Lena & 32.47 & 36.98 \\
\hline
\end{tabular}


Nirmaladevi Periyasamy and Asokan Ramasamy / Journal of Computer Science 9 (10): 1406-1413, 2013

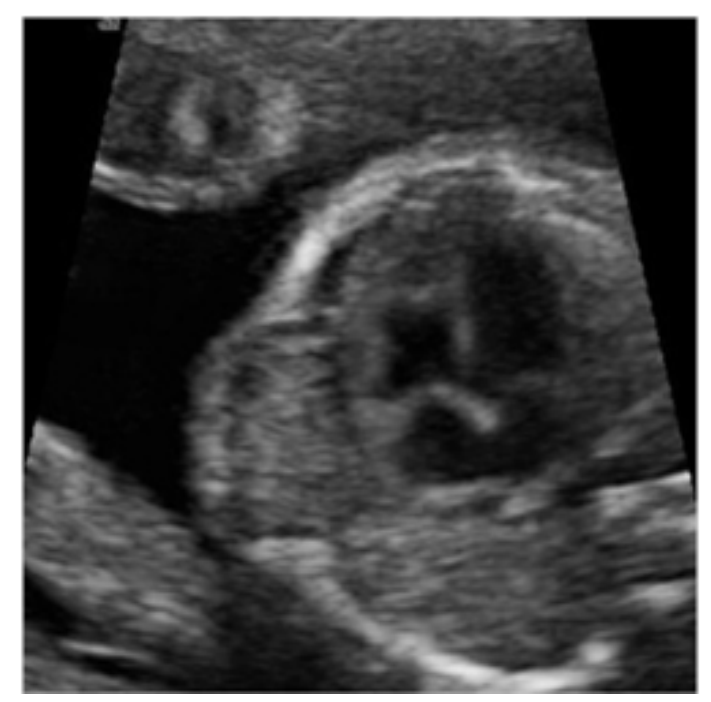

Fig. 3. Ultrasound image

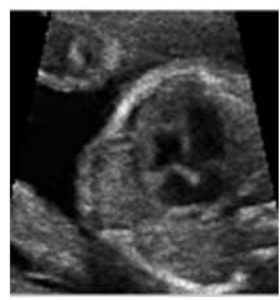

(a)

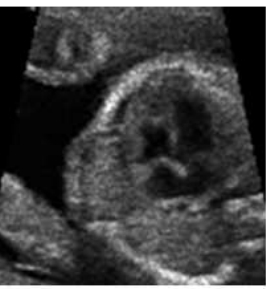

(a)

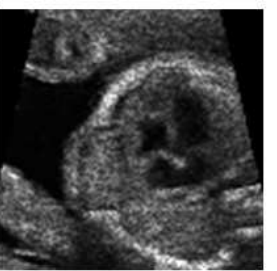

(a)

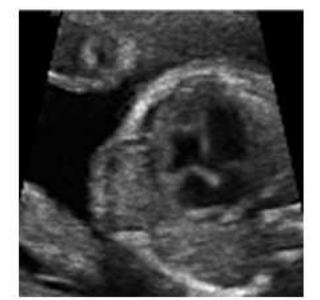

(b)

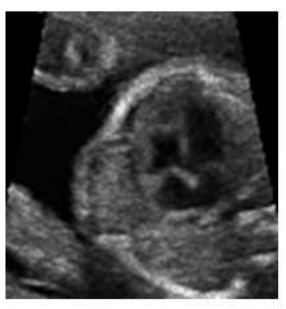

(c)

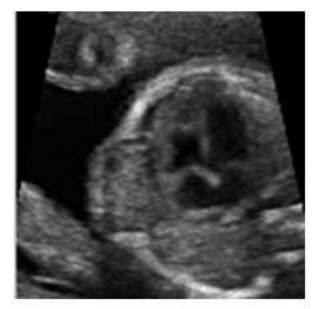

(d)

Fig. 4. Visual quality for Noise variance 0.01

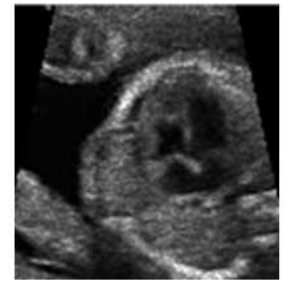

(b)

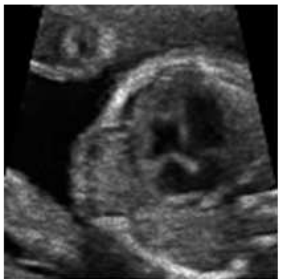

(c)

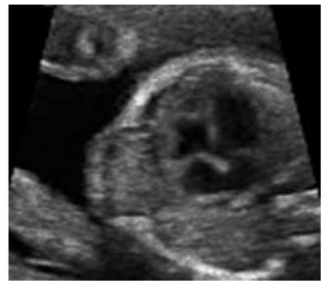

(d)

Fig. 5. Visual quality for Noise variance 0.02

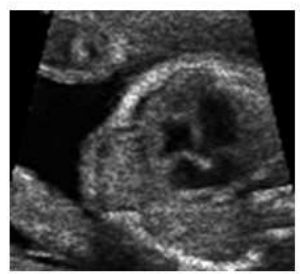

(b)

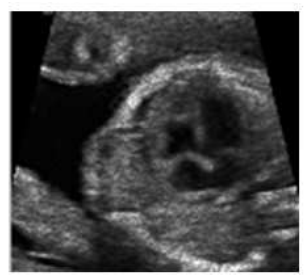

(c)

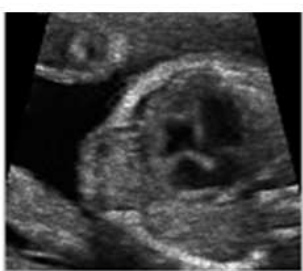

(d)

Fig. 6. Visual quality for Noise variance 0.05 


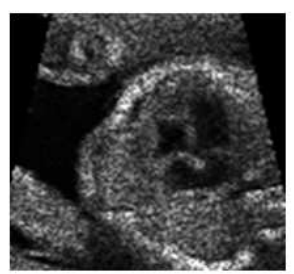

(a)

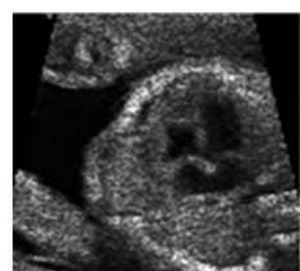

(b)

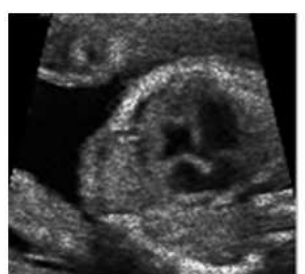

(c)

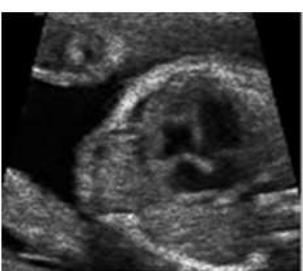

(d)

Fig. 7. Visual quality for Noise variance 0.1

Table 2. Comparison of performance measures for various noise variances

\begin{tabular}{llrrrr}
\hline Noise variance & Measures & Noisy & Soft thresholding & Adaptive with bayes threshold & MAWSF \\
\hline 0.01 & PSNR & 34.94 & 36.29 & 38.54 & 39.95 \\
& MSE & 20.83 & 15.28 & 9.11 & 6.57 \\
& SSIM & 0.96 & 0.97 & 0.98 & 0.98 \\
& ENL & 1.66 & 1.66 & 1.69 & 1.70 \\
0.04 & EPI & 0.32 & 0.36 & 0.44 & 0.46 \\
& PSNR & 31.42 & 32.46 & 34.44 & 35.66 \\
& MSE & 46.90 & 36.94 & 23.41 & 17.64 \\
& SSIM & 0.88 & 0.90 & 0.93 & 0.94 \\
& ENL & 1.53 & 1.54 & 1.66 & 1.68 \\
0.08 & EPI & 0.17 & 0.13 & 0.22 & 0.24 \\
& PSNR & 30.41 & 31.27 & 33.02 & 34.11 \\
& MSE & 59.20 & 48.52 & 32.44 & 25.25 \\
& SSIM & 0.81 & 0.83 & 0.89 & 0.90 \\
0.1 & ENL & 1.41 & 1.41 & 0.60 & 1.65 \\
& EPI & 0.12 & 0.13 & 32.57 & 0.18 \\
& PSNR & 30.11 & 30.94 & 35.98 & 33.57 \\
& MSE & 63.35 & 52.31 & 0.87 & 28.55 \\
& SSIM & 0.78 & 0.81 & 1.58 & 0.89 \\
& ENL & 1.36 & 1.36 & 0.14 & 0.16 \\
\hline
\end{tabular}

\section{DISCUSSION}

In Fig. 4-7 (a) is the noisy image, (b) is the output of Soft Thresholding, (c) is the output of Adaptive Thresholding function without exploiting intra and ainter scale dependencies and (d) is the output of MAWSF method. The adaptive wavelet shrinkage algorithm is better in removing noise as seen from Table 2. The incorporation of the proposed inter scale measure results in the preservation of important image details as seen by an increase in PSNR and other metrics from Table 2 . The enhanced adaptive thresholding function reduces the fixed bias of the soft thresholding function and hence reconstructs image in an efficient way. This can be verified with the visual quality improvement results given in Fig. 4-7. Thus in the proposed MAWSF PSNR is found to be improved upto $5 \mathrm{~dB}$ for low noise variance and to $3.4 \mathrm{~dB}$ for high noise variance. The MSE value is also reduced. Edge preservation index is also increased than the soft thresholding approach. Visual quality of the restored image is better in the proposed MAWSF approach for both high and low noise variances.

\section{CONCLUSION}

In this study an enhanced adaptive thresholding function based wavelet shrinkage in the translation invariant wavelet domain is proposed. The proposed method utilizes a sub-band adaptive threshold and also exploits inter and intra scale dependencies at various scales of decomposition of the wavelet coefficients. The denoising efficiency depends on how much correct information is conveyed from the coarser scale to its adjacent finer scale. Hence, the incorporation of the proposed inter scale measure improved the noise 
removal performance and edge preservation ability of the filter. Further improvements may be obtained by finding methods to improve the edge preservation ability.

\section{REFERENCES}

Alavandan, J. and S.S. Baboo, 2012. Enhanced speckle filters for sonar images using stationary wavelets and hybrid inter-and intra scale wavelet coefficient dependency. Global J. Comput. Sci. Technol., 12: 12-19.

Anand, S., A. Sharma and A. Girdhar, 2011. Undecimated wavelet based new threshold method for speckle noise removal in ultrasound images. Proceedings of the International Conference on Modeling, Simulation and Control, (MSC' 11), ACSIT Press, Singapore, pp: 137-145.

Dwivedi, U.D. and S.N. Singh, 2010. Enhanced detection of power-quality events using intra and interscale dependencies of wavelet coefficients. IEEE Trans. Power Delivery, 25: 358-366. DOI: 10.1109/TPWRD.2009.2027482

Hussain, S.A. and S.M. Gorashi, 2012. Image denoising based on spatial/wavelet filter using hybrid thresholding function. Int. J. Comput. Applic., 42: 513. DOI: $10.5120 / 5750-7960$
Kalavathy, S. and R.M. Suresh, 2011. Analysis of image denoising using wavelet coefficient and adaptive subband thresholding technique. Int. J. Comput. Sci., 8: 166-172.

Kaur, J., J. Kaur and M. KAUR, 2011. Survey of despeckling techniques for medical ultrasound images. Int. J. Comp. Tech. Appl., 2: 1003-1007.

Qin, X., Y. Yue, X. Dong, X. Wang and Z. Tao, 2010. An improved method of image denoising based on wavelet transform. Proceedings of the International Conference on Computer, Mechatronics, Control and Electronic Engineering, Aug. 24-26, IEEE Xplore Press, Changchun, pp: 167-170. DOI: 10.1109/CMCE.2010.5610029

Sudha, S., G.R. Suresh and R. Sukanesh, 2009. Speckle noise reduction in ultrasound images using contextbased adaptive wavelet thresholding. IETE J. Res., 55: 135-143. DOI: 10.4103/0377-2063.54905

Yang, J., R. Feng and W. Deng, 2011. A new algorithm of image denoising based on stationary wavelet multi-scale adaptive threshold. Proceedings of the International Conference on Electronic and Mechanical and Information Technology, Aug. 1214, IEEE Xplore Press, Harbin, Heilongjiang, pp: 4550-4553. DOI: 10.1109/EMEIT.2011.6024042 